\title{
Reticulospinal Systems for Tuning Motor Commands
}

\author{
Robert M. Brownstone* and Jeremy W. Chopek \\ Sobell Department of Motor Neuroscience and Movement Disorders, Institute of Neurology, University College London, \\ London, United Kingdom
}

The pontomedullary reticular formation (RF) is a key site responsible for integrating descending instructions to execute particular movements. The indiscrete nature of this region has led not only to some inconsistencies in nomenclature, but also to difficulties in understanding its role in the control of movement. In this review article, we first discuss nomenclature of the RF, and then examine the reticulospinal motor command system through evolution. These command neurons have direct monosynaptic connections with spinal interneurons and motoneurons. We next review their roles in postural adjustments, walking and sleep atonia, discussing their roles in movement activation or inhibition. We propose that knowledge of the internal organization of the RF is necessary to understand how the nervous system tunes motor commands, and that this knowledge will underlie strategies for motor functional recovery following neurological injuries or diseases.

\section{Keywords: locomotion, mesencephalic locomotor region, reticular formation, microcircuits, sleep atonia}

\section{OPEN ACCESS}

Edited by:

Brian R. Noga,

University of Miami, United States

Reviewed by:

Marie-Claude Perreault,

Emory University, United States

Stuart Baker,

Newcastle University,

United Kingdom

Joel C. Glover,

University of Oslo, Norway

*Correspondence:

Robert M. Brownstone

r.brownstone@ucl.ac.uk

Received: 23 December 2017 Accepted: 29 March 2018

Published: 18 April 2018

Citation:

Brownstone RM and Chopek JW (2018) Reticulospinal Systems for Tuning Motor Commands.

Front. Neural Circuits 12:30.

doi: 10.3389/fncir.2018.00030

\section{INTRODUCTION}

As movement is necessary for the expression of all behavior, much of the vertebrate nervous system is involved in its production. A key site for integration of descending instructions to move is the reticular formation (RF), which is situated in the brain stem and comprised of multiple nuclei. The indiscrete nature of these regions combined with disparate neuronal types within each nucleus has led not only to some inconsistencies in nomenclature, but also to difficulties in understanding RF control of movement.

The RF is comprised of different neural types including monoaminergic, cholinergic, GABA/glycinergic and glutamatergic neurons, with glutamatergic reticulospinal neurons (RSNs) forming the key descending output. Axons of these neurons extend into the spinal cord such that RSN activity can lead to a variety of motor behaviors. Inputs to RSNs descend from diverse brain regions including the telencephalon, diencephalon and cerebellum, and ascend from the spinal cord. Local circuits within RF nuclei may also contribute to their output. RSNs therefore play a role in integrating and processing these diverse inputs in order to produce effective motor behaviors. Recent reviews have addressed the role of the RF in context specific locomotion (Kim et al., 2017) and the interaction of the control of posture and locomotion (Takakusaki et al., 2016). In this review, we will discuss glutamatergic RSN systems with a focus on their organization, connectivity with spinal neurons, control of hind limb movement, and the role of these systems in recovery of

\footnotetext{
Abbreviations: DTF, dorsal tegmental field; Gi, gigantocellular reticular nucleus; GiA, gigantocellular reticular nucleus, pars alpha; GRN, gigantocellular reticular nucleus; LPGi, lateral paragigantocellular nucleus; MdV, medulla reticular formation-ventral part; medRF, medulla reticular formation; MLR, mesencephalic locomotor region; PnC, pontine reticular formation, caudal part; $\mathrm{PnO}$, pontine reticular formation, oral part; PPN, pedunculopontine nucleus; RF, reticular formation; RSN, reticulospinal neurons; SLDT, sublaterodorsal tegmental; VTF, ventral tegmental field.
} 
function after neurological injury. Additionally, we address the lack of information regarding the internal organization of RF nuclei and why this knowledge is vital for understanding how RS output is refined.

\section{RETICULAR FORMATION NOMENCLATURE}

One difficulty in determining specific functions of the RF arises from the non-discrete boundaries between nuclei and the inconsistent nomenclature in the literature. In this review, we will use the terminology as set out by Paxinos and Franklin (2008). As different investigators have used different terms, in this section we have attempted to harmonize the nomenclature through assessment of the available anatomical sections provided in publications focussed on the role of RSNs in movement.

The RF extends through the mesencephalon, pons and medulla and is traditionally divided into three columns-median, medial and lateral. In this review, we will focus on RSNs involved in limb movement, which arise from the medial column in the pons and medulla. Descending neurons from the median column arise from the raphe nuclei and are responsible for serotoninergic neuromodulation (see review: Schmidt and Jordan, 2000). The neurons of the lateral column and those in the mesencephalic RF do not project to the spinal cord.

The medial pontine RF, from rostral to caudal, comprises the nucleus reticularis pontis oralis $(\mathrm{PnO})$, from the decussation of the superior cerebellar peduncles to the trigeminal motor pool, and the nucleus reticularis pontis caudalis ( $\mathrm{PnC})$, overlapping with $\mathrm{PnO}$ and extending caudally to the facial nucleus. Situated dorsal to these nuclei are the pontine tegmental nuclei, divided into ventral (region referred to as the ventral tegmental field, or VTF), dorsal (region referred to as the dorsal tegmental field, or DTF), and lateral tegmental nuclei, as well as the sublaterodorsal tegmental (SLDT) nucleus. The dorsal tegmental nucleus extends caudally to lie dorsal to the gigantocellular reticular nucleus (GRN, vide infra), by which point the ventral and lateral tegmental nuclei merge to form the laterodorsal tegmental nucleus (LDT). In addition, rodents may have a ventral nucleus of the medial pontine RF (PnV, Liang et al., 2011) that contains a small population of RS neurons, although it has been suggested this is part of the rostral medullary GRN (see Sivertsen et al., 2016).

The rostral portion of the medial medullary RF (medRF) begins at the most caudal portion of the PnC, with the GRN lying ventral to $\mathrm{PnC}$ and extending from the facial nucleus to the obex. The GRN (sometimes referred to as Gi) is a large nucleus that contains the largest cells in the RF. Ventral to this is the pars alpha of the GRN (GiA), which merges with the Gi pars ventral (GiV) caudally. In the cat literature, the GRN is often referred to as the nucleus reticularis gigantocellularis (NRGc) or gigantocellular tegmental field (FTG), and the $\mathrm{GiA} / \mathrm{GiV}$ as the nucleus reticularis magnocellularis (NRMc) or magnocellular tegmental field (FTM, see Noga et al., 1988; Takakusaki et al., 2016), with medullary sections $9 \mathrm{~mm}$ caudal to the junction of the superior and inferior colliculi (P9) in the cat corresponding approximately to Bregma
-7 in the mouse (Paxinos and Franklin, 2008). The dorsal paragigantocellular nucleus (DPGi) is dorsal to the GRN, and the parvocellular reticular nucleus (PCRt) is dorsolateral to the GRN. The intermediate reticular zone (IRt) which contains the nucleus ambiguus, separates the GRN from the PCRt (Paxinos et al., 2012). The lateral paragigantocellular nucleus (LPGi), lies lateral to $\mathrm{GiV}$ and ventromedial to nucleus ambiguus. In the caudal medulla which lacks giant GRN neurons, the central nucleus can be subdivided into the medRF ventral part (MdV), and more cellular dorsal part $(\mathrm{MdD})$, separated by the caudal IRt and located in the caudal medulla lateral to the medial longitudinal fasciculus.

It should be noted that the borders between these regions are somewhat indistinct, necessitating a degree of caution when interpreting experimental findings involving either electrical stimulation (Mori et al., 1978; Garcia-Rill and Skinner, 1987; Takakusaki et al., 2016) or local injections (Noga et al., 1988; Takakusaki et al., 2016; Capelli et al., 2017). The comparison of histological sections between studies is helpful, but co-labeling with antibodies against choline acetyltransferase would be helpful in aligning sections based on consistent cholinergic nuclei such as motor pools.

\section{RETICULOSPINAL NEURONS ARE COMMAND NEURONS FOR MOVEMENT}

\section{RSNs Are Evolutionary Conserved Command Neurons}

The reticulospinal (RS) system is a distributed network of neurons extending from the caudal midbrain through the pons and medulla (Peterson, 1984). RSNs receive inputs from rostral motor centers and have axons that descend through the ventrolateral funiculus of the spinal cord to form synapses with spinal interneurons and motoneurons that participate in movement. As RSNs are located between higher centers that select movement and spinal cord circuits where movement is organized, RSNs may be considered as command neurons.

Command neurons are widespread across invertebrate and vertebrate species. To be classified as a command neuron, a candidate neuron must satisfy the criteria of both sufficiency and necessity for initiating a given behavior (Kupfermann and Weiss, 1978). Command neurons in the brain stem have been identified in a number of vertebrate species in which they have been shown to be involved in motor behaviors such as escape and locomotion.

Dating back more than 500 million years, the early appearing agnathans, lampreys and hagfish, developed RS command neurons called Müller cells, that have axons that cross in the brainstem and descend the length of the spinal cord to evoke swimming (Shapovalov, 1972). Perhaps the prototypical RS command neuron, though, is the Mauthner cell, initially described in teleosts which arose $\sim 310$ million years ago. Given that Mauthner cells were readily identifiable and that they share similar location, morphology, and synaptic connectivity across species, they have been well studied as command neurons (Sillar et al., 2016). Mauthner cells are likely present in lower vertebrates 
such as lamprey, but their function has mostly been studied in later-evolving fish as well as amphibians.

Two Mauthner cells, located directly opposite each other near the midline of the medulla can readily be identified in the RF based on their large cell size and location at the level of the 4th rhombomere (Sillar et al., 2016; Hildebrand et al., 2017). The axons of Mauthner cells cross in the brain stem and project through the spinal cord where they form glutamatergic, excitatory connections to large primary motoneurons and premotor excitatory interneurons (Fetcho and Faber, 1988; Faber et al., 1989; Fetcho, 1991). These interneurons have descending projections, are electrotonically coupled to large motoneurons, and form chemical synapses with small motoneurons (Fetcho, 1992). The Mauthner cell axon is also electrotonically coupled to glycinergic commissural interneurons that inhibit the large motoneurons and interneurons on the contralateral side (Yasargil and Sandri, 1990; Fetcho, 1991).

Mauthner cells receive multiple sensory inputs (Sillar et al., 2016). In response to acoustic stimuli (cf. startle response in humans below), Mauthner cells fire a single action potential (Zottoli, 1977). Based on the connectivity described above, this results in excitation of contralateral motoneurons and inhibition of ipsilateral motoneurons. This produces a fast and forceful C-start escape, arising from the initial C-bend, in which the fish moves away from the initial stimulus (Fetcho, 1992). Thus, Mauthner cell activation produces a conserved stereotypical C-start response that mediates escape (Eaton et al., 1988; Faber et al., 1989).

It is now recognized that the C-start escape response is not necessarily stereotypical, and that depending on the stimulus site and intensity, combinations of RSNs, including Mauthner cells, can produce variations of the escape response (Eaton et al., 2001). For example, it was recently demonstrated that Mauthner cells are involved in a second type of escape response, the S-start escape, when bilaterally activated (Liu and Hale, 2017). The selection of the S- vs. C-start was determined by the recruitment of segmental inhibitory neurons in spinal circuits, which would modify the response. The adaptability in behavioral responses mediated by the RS system indicates that responses to command neuron activity are variable and statedependent.

With further study, it became apparent that the Mauthner cell has multiple smaller homologoues and that they are organized in a segmental plan, similar to the segmented organization seen in invertebrates (Kimmel, 1993). It was also demonstrated that there are additional command neurons in the teleost RS system, which comprises approximately 500 neurons (Prasada Rao et al., 1987). Thus, even in these relatively early appearing teleosts, complex descending systems were present.

As supraspinal systems developed to control limbed movement, the RS system remained a dominant system mediating movement. Across species including frog, turtle, rat, cat and rhesus monkey, RSNs project to and monosynaptically excite limb motoneurons (Shapovalov, 1972). It is noteworthy that, in addition to the RS system, other descending systems such as the vestibulospinal system appeared beginning in amphibians (Shapovalov, 1972). While these other systems evolved further in reptiles, the RS system also expanded and diversified to include, for example, inhibitory RSNs. With further evolution of corticospinal including corticomotoneuronal systems in primates, the RS system persisted, producing responses in spinal motoneurons similar to those seen in fish. Thus, the RS system remains conserved though evolution (Shapovalov, 1972), and plays an important role in vertebrate, including human, movement.

In fact, the RS system was not only conserved but it flourished in evolution. For instance, compared to the 500 RSNs in the fish, there are approximately 50,000 RSNs in the mouse. Of these, approximately 19,000 are located in the GRN alone (Liang et al., 2011). Based on a comparison of EPSPs and IPSPs across species, Shapovalov concluded "the basic similarity between reticulo-motoneuronal projections across all vertebrates investigated may evidently be attributable to the common origin of the reticulospinal system, which undergoes mainly quantitative changes in the course of evolution" (Shapovalov, 1972, p. 353). In other words, as behavior became more complex, so too did the RS system. But the fundamental architecture and role of the RS system persisted across species: RSNs are large cells with dense arborizations and large fast-conducting axons that descend in the spinal cord, forming synapses with interneurons and motoneurons in multiple segments in order to produce movement.

\section{Reticulospinal Neurons Project to Spinal Interneurons and Motoneurons}

The influence of the brainstem on posture was documented by Sherrington (1898) who noted that decerebration led to "decerebrate rigidity" or hyperactivity of extensor muscles, suggesting that there is an excitatory influence from sub-cerebral structures to the spinal cord. In the 1940s Rhines and Magoun demonstrated that following decerebration, stimulation of the ventral medulla could result in the complete loss of muscle tone, and cessation of stimulation led to the immediate return of hyperextensor activity, demonstrating that the RS system had both an excitatory and inhibitory influence on postural muscles (Magoun and Rhines, 1946; Rhines and Magoun, 1946). They also demonstrated that the RS system could either facilitate or inhibit spinal reflexes in the cat (Magoun, 1944; Magoun and Rhines, 1946; Rhines and Magoun, 1946), and found that the area responsible for inhibition resided in the ventral rostral medulla (Magoun and Rhines, 1946), whereas the excitatory regions extended through the pons and medulla (Rhines and Magoun, 1946). However, Sprague and Chamber (1954) found that in intact cats, for the most part, micro-stimulation of the RF led to reciprocal (flexion-extension as well as right-left) responses in the limbs rather than generalized facilitation or inhibition. Neurons in the caudal pons and GRN form either excitatory (Grillner and Lund, 1968) or inhibitory (Llinas and Terzuolo, 1964a,b) connections with flexor or extensor hindlimb motoneurons. In addition to the hindlimb responses, RS stimulation can produce direct excitation or inhibition of neck, back, and forelimb motoneurons (Wilson and Yoshida, 1969; Wilson et al., 1970; Peterson et al., 1978). In non-human primates, activation of movement by RS stimulation is achieved by both direct and 
indirect connectivity to motoneurons (Riddle et al., 2009). Thus, RSNs produce effects that are heterogeneous and widespread in the spinal cord.

It is important to note that, in contrast to other descending systems such as the lateral vestibulospinal and corticofugal systems, the RS system is not exclusively excitatory. A proportion of RSNs descending in the MLF are inhibitory (Du Beau et al., 2012), and approximately $20 \%$ of RS synaptic contacts on propriospinal and spinal commissural interneurons in the lumbar spinal cord are inhibitory (Mitchell et al., 2016). Recently it has been shown that glycinergic neurons located in the GRN descend to the lumbar spinal cord (Valencia Garcia et al., 2018; vide infra).

Within the RF, specific regions have been found to be responsible for specific responses (Drew and Rossignol, 1990). Ipsilateral projecting pontine RSNs outnumber contralateral projecting pontine RSNs three to one and are located throughout the pontine RF whereas the contralateral projecting neurons are concentrated in the rostral pontine RF (Sivertsen et al., 2016). In neonatal mice, medial neurons in the medRF predominantly activate lateral motor column (limb-innervating) $\mathrm{MNs}$ in the lumbar spinal cord, whereas lateral medRF neurons predominantly activate medial motor column (axial muscle-innervating) MNs (Szokol et al., 2008). In both cases, these actions may be mediated through polysynaptic pathways mediated by ipsilateral and contralateral descending spinal commissural interneurons (Szokol et al., 2011; Perreault and Glover, 2013).

Microstimulation within the RF can result in movement across multiple joints or limbs. For example, stimulation that produced forelimb movement was regularly accompanied by movement of the head or hindlimbs (Drew and Rossignol, 1990). Furthermore, homologous to Mauthner cell responses, motoneuron responses were not restricted to one side: microstimulation could evoke ipsilateral flexion and contralateral extension of the either the fore or hind limbs (Drew and Rossignol, 1990). These evoked movements are consistent with the axonal projections of RSNs, with $85 \%$ of axons extending to both the cervical and lumbar enlargements, and, at least in the cervical enlargement, axons projecting bilaterally (Peterson et al., 1975). Thus, RSNs have divergent spinal projections and are involved in multi-joint, multi-limb motor commands. In summary, the RS system in the mammal is functionally organized and acts to coordinate multiple movements including excitation and inhibition across joints and across limbs.

\section{medRF Reticulospinal Neurons and Locomotion}

The rhythm and pattern of locomotion are produced by spinal cord circuits in response to descending commands (Graham Brown, 1911; Jankowska et al., 1967). RF locomotor command neurons are activated by an upstream center called the mesencephalic locomotor region (MLR). MLR stimulation produces locomotion in decerebrate cats (Shik et al., 1966), rats (Skinner and Garcia-Rill, 1984), and mice (Roseberry et al., 2016; Stecina, 2017; Caggiano et al., 2018) through a pathway mediated by medRF RSNs (Noga et al., 2003). The medRF has been shown to be crucial for locomotion in a number of other species as well, including lamprey (McClellan and Grillner, 1984), ducks and geese (Steeves et al., 1987) and guinea pigs (Marlinskii and Voitenko, 1992). In the cat, medRF RSNs in the GRN are necessary for MLR-evoked locomotion, and are sufficient to induce locomotor activity through electrical (Jordan et al., 2008) or chemical stimulation (Noga et al., 1988). These "locomotor" medRF RSNs also receive input from the contralateral cerebellar locomotor region (Mori et al., 1998) and from the ipsilateral lateral hypothalamic area, termed the subthalamic locomotor region (Sinnamon and Stopford, 1987). Given the multitude of locomotor-related inputs, Orlovskii (1970) stated that "the invariable mediating link for initiating locomotion is the reticulospinal system."

Locomotor medRF RSNs are fast conducting (Orlovskii, 1970; Degtyarenko et al., 1998; Noga et al., 2003) and glutamatergic (Douglas et al., 1993; Jordan et al., 2008). They are phasically active during spontaneous locomotion in thalamic cats (Shimamura et al., 1982; Shimamura and Kogure, 1983), treadmill locomotion in unrestrained cats (Drew et al., 1986; Matsuyama and Drew, 2000), and spontaneous or MLR-evoked fictive locomotion in the absence of movement related feedback in decerebrate cats (Perreault et al., 1993). Locomotion-inducing RSN axons descend in the ventrolateral funiculus (Steeves and Jordan, 1980), may innervate the ipsi- or contra-lateral spinal cord (Matsuyama et al., 1988, 1999), and are known to innervate commissural interneurons in the lumbar spinal cord (Matsuyama et al., 2004; Jankowska, 2008). That is, RSNs in the medRF GRN provide extensive innervation to bilateral spinal motor regions, and seemingly fulfil the criteria of command neurons for locomotion.

While RSNs thus integrate inputs from multiple higher centers in order to send commands to the spinal cord, there may also be local processing of commands within the RF. Neurons within the RF have heterogeneous characteristics, leading to the suggestion that there may be local circuit processing of reticulospinal commands (Shimamura et al., 1980), but this has not been explored in detail. For example, a subset of neurons in this region in lamprey (Einum and Buchanan, 2004; Buchanan, 2011) and cat (Shimamura et al., 1980, 1982) receive inputs from the spinal cord. In the cat, it has been suggested that the phasic activity of RSNs during fictive locomotion may arise from inputs from the spinal cord (Perreault et al., 1993). This hypothesis is supported by the demonstrated phase-dependent modulation of RSN activity in response to cutaneous stimulation during locomotion (Drew et al., 1996). How these ascending inputs are processed and how they might affect the descending commands is not yet clear.

There is also evidence that some RSNs receive neuromodulatory input (Takakusaki et al., 1993a), indicating that their activity may be state dependent (Takakusaki et al., 2016). While there has been focus on modulation of RSNs by acetylcholine (Takakusaki et al., 1993b; Le Ray et al., 2010), serotonin also may play a role (Takakusaki et al., 1993a). Given the importance of serotoninergic activity in locomotion (Schmidt and Jordan, 2000), it is possible that this modulatory 
system is involved in both brain stem and spinal circuits for locomotion.

Pathways for different types of locomotor activity, such as escape vs. exploration, may differ (Sinnamon, 1993; Jordan, 1998). The MLR is comprised primarily of the cuneiform nucleus (Jordan, 1998), but recent evidence in the mouse suggests that while higher speed locomotion is mediated by the cuneiform nucleus, exploratory activity is initiated by a region just ventral to this, the pedunculopontine nucleus (PPN, Caggiano et al., 2018). The PPN has diverse projections to RF nuclei (Gi, $\mathrm{GiV}, \mathrm{GiA}, \mathrm{LPGi}, \mathrm{MdV}$ ), whereas the cuneiform projections are more restricted (LPGi, GiV, GiA but not the Gi or MdV, Caggiano et al., 2018). While cat studies have pointed towards the medial regions of the medRF as the command for locomotion (Noga et al., 2003), there has been a recent suggestion that in the mouse, LPGi activation is necessary for higher speed locomotion (Capelli et al., 2017). This suggests that there may be species differences in descending commands, a difference in the state of the circuits being studied across experiments, or that locomotor activity is initiated via indirect pathways in some experiments (see Noga et al., 2003). In summary, it is clear across multiple species that the medRF plays a key role in integrating and processing instructions for locomotion from more rostral centers.

In addition to their well-defined role in initiating locomotion, RSNs have recently been implicated in stopping locomotion. Optogenetic activation of a subset of glutamatergic neurons defined by expression of the transcription factor Chx10 located in the PnC and GRN regions can lead to cessation of locomotor activity in the mouse (Bouvier et al., 2015) and lamprey (Juvin et al., 2016). Three activity patterns in RSNs were seen in this region during swimming in lamprey: those that fired exclusively at the onset of swimming ("start" RSNs), those that fired throughout the swimming bout ("start and maintain" RSNs), and those that fired prior to the termination of locomotion ("stop" RSNs; Juvin et al., 2016). This indicates that there is a diversity of neuronal function within the RSN. In the mouse and possibly lamprey, the behavioral response from the stop RSNs is likely mediated by spinal inhibitory neurons targeted by these descending glutamatergic RSNs (Bouvier et al., 2015; Juvin et al., 2016).

The ability of RSNs in the medRF/GRN to act as command neurons for a variety of behaviors in addition to starting and stopping locomotion is highlighted by its involvement in a multitude of tasks ranging from, for example, maintaining posture during walking to atonia during sleep (vide infra).

\section{Interaction of Reticulospinal Systems for Locomotion and Posture}

In the late 1970s, it was shown that postural adjustments associated with locomotion originated in the caudal portion of the pontine RF (see Mori, 1987). Stimulation of the dorsal area of the caudal pontine tegmental field (DTF) resulted in the relaxation of extensor muscles, whereas stimulation of the ventral area of the caudal pontine tegmental field (VTF) resulted in activation or an increase in extensor muscle tone. More recently, it has been shown that injections of cholinergic and serotonergic agonists into the $\mathrm{PnO}$ resulted in atonia and hypertonia respectively. These effects were mediated through medRF RSNs, with atonia-related RSNs located in the dorsomedial part of the medRF and hypertonus-related RSNs located in the ventromedial part of the medRF (Takakusaki et al., 2016). Thus it is clear that RSNs, through excitation and inhibition of spinal circuits, regulate muscle tone (for review see Takakusaki et al., 2016).

Extensor muscle tone is necessary for locomotion as there must be adequate force production to support the body and propel limbs forward (Mori et al., 1978, 1982). The interplay of posture and locomotion was demonstrated during MLR evoked locomotion, when stimulation of the DTF halted locomotion and stimulation of the VTF resulted in enhanced locomotor activity or a change in gait pattern (Mori et al., 1978). In freely moving cats with electrodes implanted in the DTF or VTF and the MLR, DTF stimulation during walking (naturally or MLR induced) led to sequential postural reduction to a sitting then prone position, and VTF stimulation when lying led to a rise to stance followed by locomotion (Mori et al., 1986).

The co-expression of postural tone and locomotion is likely due to shared neural pathways. For example, in addition to projecting to locomotor neurons in the GRN, the MLR also projects to postural areas (DTF and VTF). About $70 \%$ of DTF neurons are activated by MLR stimulation, with most being tonically active but some demonstrating rhythmic activity (Kawahara et al., 1985). Thus the MLR through two DTF pathways may participate in both tonic regulation of posture for locomotion and phasic regulation contributing to step cycle timing.

\section{Reticulospinal Neurons and Reaching}

RSNs are involved in movements other than posture and locomotion. For example, some RSNs in the cat are active during the preparatory phase in anticipation of reaching, while others are active during the actual reaching task (Schepens, 2004). Anatomical evidence using viral tracing has demonstrated glutamatergic RSNs in the MdV that form synapses exclusively with forelimb and not hindlimb MNs, unlike other neurons, including those in the GRN, which form synapses with both forelimbs and hindlimb MNs (Esposito et al., 2014). Furthermore, these synapses are motor pool specific, appearing on biceps but not triceps motoneurons. Inhibition of these RSNs resulted in decreased ability of the mice to accurately reach and grasp during a food pellet challenge. Because these RSNs are not involved in hindlimb movement, we will not consider them further here.

\section{Reticulospinal Neurons and Sleep Atonia}

While descending commands can stop locomotion (vide supra), the prototypical "stop" command is that which produces sleep atonia, in which limb muscle tone is curtailed to prevent movement during REM sleep (Saper et al., 2010). This atonic command originates in the SLDT nucleus, which contains glutamatergic neurons that project both to GRN and to the spinal cord (Sastre et al., 1981; Chase et al., 1986; Saper et al., 2010). Both of these targets contribute to sleep atonia, but whether 
the direct descending command from the GRN is inhibitory and/or whether its inhibitory effects are mediated by spinal interneurons is not clear (Fuller et al., 2007; Arrigoni et al., 2016). Recent evidence, however, suggests that there is descending inhibitory input that produces sleep atonia (Valencia Garcia et al., 2018).

In cats, lesions to the subcoeruleus region (analogous to the SLDT) result in behavior in which the cats appear to act out their dreams during sleep (Mouret et al., 1967; Henley and Morrison, 1974). Similarly, lesions to the SLDT in rodents led to complex motor behaviors during REM sleep (Lu et al., 2006). These behaviors are akin to REM behavior disorder (RBD) in humans (Schenck and Mahowald, 1995). That RBD can be localized to the SLDT has been shown in a case report of a woman with a lesion in this region who suffered from RBD and somnambulism (Limousin et al., 2009). Interestingly, RSNs that are associated with specific movements during waking are also active during REM sleep (Siegel et al., 1981). This suggests that extraneous movements during RBD are produced by the same neurons as during wakefulness. RBD is perhaps an extreme example of pathological RSN circuit selection. Other examples could include other disorders affecting movement selection such as dystonic syndromes.

\section{INTRINSIC ORGANIZATION OF THE RETICULAR FORMATION}

Clearly, rostral centers must activate different populations of RSNs in order to produce selected movements. Given that there can be net inhibitory (sleep atonia) and net excitatory (locomotion) effects produced by RSNs, we propose that movement selection circuits activate smaller ensembles or microcircuits of RSNs in order to facilitate specific movements whilst inhibiting other movements. This concept is not unlike that of the direct and indirect pathways of the basal ganglia that cooperate in movement selection (Cui et al., 2013). This example also demonstrates that the control of moving and not moving are intimately intertwined, and that neurological disorders that alter either one or the other of these two classes of microcircuits can lead to significant impairment in quality of life. How these microcircuits interact with each other, however, is yet to be defined.

What determines the tuning of appropriate commands? As RSNs are involved in diverse movements and comprise a subset of RF neurons, it would be prudent to ask whether RSNs simply integrate and relay inputs from higher brain centers to the spinal cord, or whether local microcircuits participate in refining motor commands.

Whilst our knowledge of the functional and topographical organization of RSNs is increasing, little is known about the internal organization of the RF. Some neurons within these nuclei are inhibitory, presumably interneurons (Sivertsen et al., 2016), but there is little information about local connectivity. Several modeling studies have addressed this lack of understanding of the internal circuitry. One proposal is that the RF is a "small-world network" in which dense connectivity within small groups of nodes is responsible for appropriate action selection (Humphries et al., 2006). Individual nodes would comprise small diameter local interneurons, most likely inhibitory, that project medially and laterally and connect with larger projecting cells (likely RSNs) within their own node. Node to node connectivity would be via axon collaterals of the large projecting neurons which would form synapses at relatively short distances, allowing for nearby nodes to activate quickly and concurrently (Humphries et al., 2007).

Experimental data to support this model are in short supply to date. But it is clear that knowledge of the internal organization of the RF is critical to understand how the RF and RSNs are selected to produce a wide array of finely tuned motor commands.

\section{RETICULOSPINAL NEURONS IN HUMANS AND THEIR ROLE IN RECOVERY OF MOTOR FUNCTION}

The corticofugal system plays an important role in human movement (Lemon, 2008). Axon collaterals from descending corticospinal neurons have diffuse projections, including to the RF (Kita and Kita, 2012). It is of interest that, although there was a deficit in fractionated finger movements in monkeys following lesions of these tracts below the level of the collaterals to the $\mathrm{RF}$, other movements including climbing and running persisted (Lawrence and Kuypers, 1968a,b). Furthermore, monosynaptic corticomotoneuronal synapses are not necessary for hand movements in non-human primates, which improved over time following pyramidal tract lesions (Sasaki et al., 2004). Similarly, rare reports of sectioning these tracts in humans have demonstrated initial hemiparesis followed by recovery, ultimately leaving patients with a relative lack of movement deficit (Bucy et al., 1964). These data suggest that other systems such as RSNs can play a prominent role in movement in primates, including humans.

RS systems have been studied in humans via the startle reflex. This reflex response is seen across species and been shown to originate in the caudal brainstem-most likely the PnC (Hammond, 1973; Leitner et al., 1980; Davis et al., 1982). The startle reflex is seen in response to a loud acoustic stimulus (cf. Mauthner cells, above), and is stereotypically comprised of activation of the sternocleidomastoid muscle, followed by cranial nerve innervated muscles and rectus abdominus, and finally forelimb and hindlimb muscles (Rothwell, 2006). Evidence for a brainstem origin for this response has come from electrophysiological studies. When the instruction to start voluntary movement was combined with a loud auditory cue, the time to movement onset was significantly reduced (Rothwell, 2006). For example, the onset to volitional arm movement was found to be $150 \mathrm{~ms}$, whereas arm movement initiated by the startle response can occur in $80 \mathrm{~ms}$. This reduced latency has also been associated with an increased rate of force development, as well as increased force output (Anzak et al., 2011). Moreover, in more complex movements involving sequential activation of agonist and antagonist muscles, auditory stimulation sped up the activation of movement without affecting the pattern of muscle 
activity (Valls-Solé et al., 1999). Together, these data all point towards a subcortical, and more specifically RS, origin for startle reflexes.

The startle response in humans integrates with voluntary movements and may thus facilitate a potential therapeutic approach for regaining volitional movement post neurological insults to the cortex such as stroke (Rothwell, 2006) or to enhance motor performance in diseases such as Parkinson's disease (Anzak et al., 2016). In stroke patients, when the startle response was combined with voluntary movement, the latency to EMG response was shorter and the response was much larger than when either was performed alone, leading to the suggestion that detail of voluntary movement can be stored in the brainstem and can be accessed to produce movement in the absence of descending inputs from the cerebral cortex (Rothwell, 2006).

Given that RSNs are important in movement, and that many neurological injuries occur above the level of the RF, it is reasonable to ask whether the RS system could be a therapeutic target for therapies aimed at recovery of motor function in neurological diseases and injuries such as stroke (Baker et al., 2015). Data from non-human primates supports this concept (Zaaimi et al., 2012). Presumably, intentional signals from higher centers would still need to reach the RF, but as noted above, there are many supramedullary inputs that converge on RSNs. For example, following unilateral stroke, there are non-crossed cortico-reticular inputs that could possibly harness the unaffected ipsilateral cortex to promote RF plasticity and motor functional recovery (Jankowska and Edgley, 2006). The RS system is well connected to play a key role in recovery from motor deficits, as it projects to all levels of the spinal cord including to key movement circuits.

Such RS-mediated functional recovery need not be limited to axial movement. Although initially proposed to be important for axial and proximal muscles, it is becoming clear from studies in non-human primates that RSNs are also involved in hand function (Riddle et al., 2009), and could thus be a

\section{REFERENCES}

Anzak, A., Tan, H., Pogosyan, A., and Brown, P. (2011). Doing better than your best: loud auditory stimulation yields improvements in maximal voluntary force. Exp. Brain Res. 208, 237-243. doi: 10.1007/s00221-010-2474-1

Anzak, A., Tan, H., Pogosyan, A., Khan, S., Javed, S., Gill, S. S., et al. (2016). Subcortical evoked activity and motor enhancement in Parkinson's disease. Exp. Neurol. 277, 19-26. doi: 10.1016/j.expneurol.2015.12.004

Arrigoni, E., Chen, M. C., and Fuller, P. M. (2016). The anatomical, cellular and synaptic basis of motor atonia during rapid eye movement sleep. J. Physiol. 594, 5391-5414. doi: 10.1113/JP271324

Baker, S. N. (2011). The primate reticulospinal tract, hand function and functional recovery. J. Physiol. 589, 5603-5612. doi: 10.1113/jphysiol.2011.215160

Baker, S. N., and Perez, M. A. (2017). Reticulospinal contributions to gross hand function after human spinal cord injury. J. Neurosci. 37, 9778-9784. doi: 10.1523/JNEUROSCI.3368-16.2017

Baker, S. N., Zaaimi, B., Fisher, K. M., Edgley, S. A., and Soteropoulos, D. S. (2015). Pathways mediating functional recovery. Prog. Brain Res. 218, 389-412. doi: 10.1016/bs.pbr.2014.12.010

Bouvier, J., Caggiano, V., Leiras, R., Caldeira, V., Bellardita, C., Balueva, K., et al. (2015). Descending command neurons in the brainstem that halt locomotion. Cell 163, 1191-1203. doi: 10.1016/j.cell.2015.10.074 substrate for functional recovery of hand movements following neurological injury (Baker, 2011). In support of this possibility, recent evidence from humans points to the RS system as being involved in gross hand function after spinal cord injury (Baker and Perez, 2017).

To promote RSN-mediated recovery of motor function, however, several challenges exist. It will be necessary to understand the functional organization of motor command circuits in the RF, including how nodes or microcircuits in the RF are engaged to produce movement, whether individual microcircuits participate in more than one motor behavior, how individual microcircuits are recruited by rostral circuits, whether these circuits are under neuromodulatory control, and whether "on" movement and "off" movement microcircuits are simultaneously recruited to refine motor commands. Understanding these concepts could form the foundation for future therapeutic strategies.

Ultimately, it is clear that our focus in understanding the RF should include not only an "integrate and relay" function, but also an understanding of the internal organization and processing that controls RSNs such that a great versatility of movements can be produced. This understanding will lead not only to improved knowledge of how we move, but potentially also to new experimental paradigms to understand motor system changes in injury and disease, and ultimately to the development of new therapeutic strategies aimed at improving motor functional recovery.

\section{AUTHOR CONTRIBUTIONS}

$\mathrm{RB}$ and JC conceived and wrote the manuscript.

\section{FUNDING}

RB was supported by Wellcome Trust (grant no. 110193) and Brain Research UK.

Buchanan, J. T. (2011). Spinal locomotor inputs to individually identified reticulospinal neurons in the lamprey. J. Neurophysiol. 106, 2346-2357. doi: $10.1152 /$ jn. 01100.2010

Bucy, P. C., Keplinger, J. E., and Siqueira, E. B. (1964). Destruction of the 'pyramidal tract' in man. J. Neurosurg. 21, 285-298.

Caggiano, V., Leiras, R., Goñi-Erro, H., Masini, D., Bellardita, C., Bouvier, J., et al. (2018). Midbrain circuits that set locomotor speed and gait selection. Nature 553, 455-460. doi: 10.1038/nature25448

Capelli, P., Pivetta, C., Soledad Esposito, M., and Arber, S. (2017). Locomotor speed control circuits in the caudal brainstem. Nature 551, 373-377. doi: 10.1038 /nature24064

Chase, M. H., Morales, F. R., Boxer, P. A., Fung, S. J., and Soja, P. J. (1986). Effect of stimulation of the nucleus reticularis gigantocellularis on the membrane potential of cat lumbar motoneurons during sleep and wakefulness. Brain Res. 386, 237-244. doi: 10.1016/0006-8993(86) 90160-5

Cui, G., Jun, S. B., Jin, X., Pham, M. D., Vogel, S. S., Lovinger, D. M., et al. (2013). Concurrent activation of striatal direct and indirect pathways during action initiation. Nature 494, 238-242. doi: 10.1038/nature11846

Davis, M., Gendelman, D. S., Tischler, M. D., and Gendelman, P. M. (1982). A primary acoustic startle circuit: lesion and stimulation studies. J. Neurosci. 2, 791-805. 
Degtyarenko, A. M., Simon, E. S., and Burke, R. E. (1998). Locomotor modulation of disynaptic EPSPs from the mesencephalic locomotor region in cat motoneurons. J. Neurophysiol. 80, 3284-3296. doi: 10.1152/jn.1998. 80.6.3284

Douglas, J. R., Noga, B. R., Dai, X., and Jordan, L. M. (1993). The effects of intrathecal administration of excitatory amino-acid agonists and antagonists on the initiation of locomotion in the adult cat. J. Neurosci. 13, 990-1000.

Drew, T., Cabana, T., and Rossignol, S. (1996). Responses of medullary reticulospinal neurones to stimulation of cutaneous limb nerves during locomotion in intact cats. Exp. Brain Res. 111, 153-168. doi: $10.1007 / \mathrm{bf} 00227294$

Drew, T., Dubuc, R., and Rossignol, S. (1986). Discharge patterns of reticulospinal and other reticular neurons in chronic, unrestrained cats walking on a treadmill. J. Neurophysiol. 55, 375-401. doi: 10.1152/jn.1986.55.2.375

Drew, T., and Rossignol, S. (1990). Functional organization within the medullary reticular formation of intact unanesthetized cat. II. Electromyographic activity evoked by microstimulation. J. Neurophysiol. 64, 782-795. doi: 10.1152/jn.1990. 64.3 .782

Du Beau, A., Shakya Shrestha, S., Bannatyne, B. A., Jalicy, S. M., Linnen, S., and Maxwell, D. J. (2012). Neurotransmitter phenotypes of descending systems in the rat lumbar spinal cord. Neuroscience 227, 67-79. doi: 10.1016/j. neuroscience.2012.09.037

Eaton, R. C., DiDomenico, R., and Nissanov, J. (1988). Flexible body dynamics of the goldfish C-start: implications for reticulospinal command mechanisms. J. Neurosci. 8, 2758-2768.

Eaton, R. C., Lee, R. K. K., and Foreman, M. B. (2001). The Mauthner cell and other identified neurons of the brainstem escape network of fish. Prog. Neurobiol. 63, 467-485. doi: 10.1016/s0301-0082(00)00047-2

Einum, J. F., and Buchanan, J. T. (2004). Reticulospinal neurons receive direct spinobulbar inputs during locomotor activity in lamprey. J. Neurophysiol. 92, 1384-1390. doi: 10.1152/jn.00625.2003

Esposito, M. S., Capelli, P., and Arber, S. (2014). Brainstem nucleus MdV mediates skilled forelimb motor tasks. Nature 508, 351-356. doi: 10.1038/nature13023

Faber, D. S., Fetcho, J. R., and Korn, H. (1989). Neuronal networks underlying the escape response in goldfish. Ann. N Y Acad. Sci. 563, 11-33. doi: 10.1111/j. 1749-6632.1989.tb42187.x

Fetcho, J. R. (1991). Spinal network of the Mauthner cell. Brain Behav. Evol. 37, 298-316. doi: 10.1159/000316094

Fetcho, J. R. (1992). The spinal motor system in early vertebrates and some of its evolutionary changes. Brain Behav. Evol. 40, 82-97. doi: 10.1159/000113905

Fetcho, J. R., and Faber, D. S. (1988). Identification of motoneurons and interneurons in the spinal network for escapes initiated by the mauthner cell in goldfish. J. Neurosci. 8, 4192-4213.

Fuller, P. M., Saper, C. B., and Lu, J. (2007). The pontine REM switch: past and present. J. Physiol. 584, 735-741. doi: 10.1113/jphysiol.2007.140160

Garcia-Rill, E., and Skinner, R. D. (1987). The mesencephalic locomotor region. I. Activation of a medullary projection site. Brain Res. 411, 1-12. doi: 10.1016/0006-8993(87)90675-5

Graham Brown, T. (1911). The intrinsic factors in the act of progression in the mammal. Proc. R. Soc. B Biol. Sci. 84, 308-319. doi: 10.1098/rspb.1911.0077

Grillner, S., and Lund, S. (1968). The origin of a descending pathway with monosynaptic action on flexor motoneurones. Acta Physiol. Scand. 74, 274-284. doi: 10.1111/j.1748-1716.1968.tb04236.x

Hammond, G. R. (1973). Lesions of pontine and medullary reticular formation and prestimulus inhibition of the acoustic startle reaction in rats. Physiol. Behav. 10, 239-243. doi: 10.1016/0031-9384(73)90304-1

Henley, K., and Morrison, A. R. (1974). A re-evaluation of the effects of lesions of the pontine tegmentum and locus coeruleus on phenomena of paradoxical sleep in the cat. Acta Neurobiol. Exp. 34, 215-232.

Hildebrand, D. G. C., Cicconet, M., Torres, R. M., Choi, W., Quan, T. M., Moon, J., et al. (2017). Whole-brain serial-section electron microscopy in larval zebrafish. Nature 545, 345-349. doi: 10.1038/nature22356

Humphries, M. D., Gurney, K., and Prescott, T. J. (2006). The brainstem reticular formation is a small-world, not scale-free, network. Proc. R. Soc. B Biol. Sci. 273, 503-511. doi: 10.1098/rspb.2005.3354

Humphries, M. D., Gurney, K., and Prescott, T. J. (2007). Is there a brainstem substrate for action selection? Philos. Trans. R. Soc. London Ser. B Biol. Sci. 362, 1627-1639. doi: 10.1098/rstb.2007.2057
Jankowska, E. (2008). Spinal interneuronal networks in the cat: elementary components. Brain Res. Rev. 57, 46-55. doi: 10.1016/j.brainresrev.2007.06.022

Jankowska, E., and Edgley, S. A. (2006). How can corticospinal tract neurons contribute to ipsilateral movements? A question with implications for recovery of motor functions. Neuroscientist 12, 67-79. doi: 10.1177/1073858405283392

Jankowska, E., Jukes, M., Lund, S., and Lundberg, A. (1967). The effect of DOPA on the spinal cord. 6. Half-centre organization of interneurones transmitting effects from the flexor reflex afferents. Acta Physiol. Scand. 70, 389-402. doi: 10.1111/j.1748-1716.1967.tb03637.x

Jordan, L. M. (1998). Initiation of locomotion in mammals. Ann. N Y Acad. Sci. 860, 83-93. doi: 10.1111/j.1749-6632.1998.tb09040.x

Jordan, L. M., Liu, J., Hedlund, P. B., Akay, T., and Pearson, K. G. (2008). Descending command systems for the initiation of locomotion in mammals. Brain Res. Rev. 57, 183-191. doi: 10.1016/j.brainresrev.2007.07.019

Juvin, L., Grätsch, S., Trillaud-Doppia, E., Gariépy, J. F., Büschges, A., and Dubuc, R. (2016). A specific population of reticulospinal neurons controls the termination of locomotion. Cell Rep. 15, 2377-2386. doi: 10.1016/j.celrep.2016. 05.029

Kawahara, K., Mori, S., Tomiyama, T., and Kanaya, T. (1985). Discharges of neurons in the midpontine dorsal tegmentum of mesencephalic cat during locomotion. Brain Res. 341, 377-380. doi: 10.1016/0006-8993(85) 91078-9

Kim, L. H., Sharma, S., Sharples, S. A., Mayr, K. A., Kwok, C. H. T., and Whelan, P. J. (2017). Integration of descending command systems for the generation of context-specific locomotor behaviors. Front. Neurosci. 11:581. doi: 10.3389/fnins.2017.00581

Kimmel, C. B. (1993). Patterning the brain of the zebrafish embryo. Annu. Rev. Neurosci. 16, 707-732. doi: 10.1146/annurev.neuro.16.1.707

Kita, T., and Kita, H. (2012). The subthalamic nucleus is one of multiple innervation sites for long-range corticofugal axons: a single-axon tracing study in the rat. J. Neurosci. 32, 5990-5999. doi: 10.1523/JNEUROSCI.5717-11.2012

Kupfermann, I., and Weiss, K. R. (1978). The command neuron concept. Behav. Brain Sci. 1, 3-10. doi: 10.1017/s0140525x00059057

Lawrence, D. G., and Kuypers, H. G. J. M. (1968a). The functional organization of the motor system in the monkey: I. The effects of bilateral pyramidal lesions. Brain 91, 1-14. doi: 10.1093/brain/91.1.1

Lawrence, D. G., and Kuypers, H. G. J. M. (1968b). The functional organization of the motor system in the monkey: II. The effects of lesions of the descending brain-stem pathways. Brain 91, 15-36. doi: 10.1093/brain/91.1.15

Leitner, D. S., Powers, A. S., and Hoffman, H. S. (1980). The neural substrate of the startle response. Physiol. Behav. 25, 291-297. doi: 10.1016/00319384(80)90219-x

Lemon, R. N. (2008). Descending pathways in motor control. Annu. Rev. Neurosci. 31, 195-218. doi: 10.1146/annurev.neuro.31.060407.125547

Le Ray, D., Juvin, L., Boutin, T., Auclair, F., and Dubuc, R. (2010). A neuronal substrate for a state-dependent modulation of sensory inputs in the brainstem. Eur. J. Neurosci. 32, 53-59. doi: 10.1111/j.1460-9568.2010.07276.x

Liang, H., Paxinos, G., and Watson, C. (2011). Projections from the brain to the spinal cord in the mouse. Brain Struct. Funct. 215, 159-186. doi: 10.1007/s00429-010-0281-x

Limousin, N., Dehais, C., Gout, O., Héran, F., Oudiette, D., and Arnulf, I. (2009). A brainstem inflammatory lesion causing REM sleep behavior disorder and sleepwalking (parasomnia overlap disorder). Sleep Med. 10, 1059-1062. doi: 10.1016/j.sleep.2008.12.006

Liu, Y. C., and Hale, M. E. (2017). Local spinal cord circuits and bilateral mauthner cell activity function together to drive alternative startle behaviors. Curr. Biol. 27, 697-704. doi: 10.1016/j.cub.2017.01.019

Llinas, R., and Terzuolo, C. (1964a). Mechanisms of supraspinal actions upon spinal cord activities. Reticular inhibitory mechanisms on alphaextensor motoneurons. J. Neurophysiol. 27, 579-591. doi: 10.1152/jn.1964.27. 4.579

Llinas, R., and Terzuolo, C. (1964b). Mechaniss of supraspinal actions upon spinal cord activities. Reticular inhibitory mechanisms upon flexor motoneurons. J. Neurophysiol. 28, 413-422. doi: 10.1152/jn.1965.28.2.413

Lu, J., Sherman, D., Devor, M., and Saper, C. B. (2006). A putative flip-flop switch for control of REM sleep. Nature 441, 589-594. doi: 10.1038/nature04767

Magoun, H. (1944). Bulbar inhibition and facilitation of motor activity. Science 100, 549-550. doi: 10.1126/science.100.2607.549 
Magoun, H., and Rhines, R. (1946). An inhibitory mechanism in the bulbar reticular formation. J. Neurophysiol. 9, 165-171. doi: 10.1152/jn.1946.9.3.165

Marlinskii, V. V., and Voitenko, L. P. (1992). Participation of the medial reticular formation of the medulla oblongata in the supraspinal control of locomotor and postural activities in the guinea pig. Neurosci. Behav. Physiol. 22, 336-342. doi: $10.1007 / \mathrm{bf} 01182876$

Matsuyama, K., and Drew, T. (2000). Vestibulospinal and reticulospinal neuronal activity during locomotion in the intact cat. II. Walking on an inclined plane. J. Neurophysiol. 84, 2257-2276. doi: 10.1152/jn.2000.84.5.2257

Matsuyama, K., Mori, F., Kuze, B., and Mori, S. (1999). Morphology of single pontine reticulospinal axons in the lumbar enlargement of the cat: a study using the anterograde tracer PHA-L. J. Comp. Neurol. 410, 413-430. doi: 10.1002/(sici)1096-9861(19990802)410:3<413::aid-cne5>3.0.co;2-q

Matsuyama, K., Nakajima, K., Mori, F., Aoki, M., and Mori, S. (2004). Lumbar commissural interneurons with reticulospinal inputs in the cat: morphology and discharge patterns during fictive locomotion. J. Comp. Neurol. 474, 546-561. doi: 10.1002/cne.20131

Matsuyama, K., Ohta, Y., and Mori, S. (1988). Ascending and descending projections of the nucleus reticularis gigantocellularis in the cat demonstrated by the anterograde neural tracer, Phaseolus vulgaris leucoagglutinin (PHA-L). Brain Res. 460, 124-141. doi: 10.1016/0006-8993(88)91212-7

McClellan, A. D., and Grillner, S. (1984). Activation of 'fictive swimming' by electrical microstimulation of brainstem locomotor regions in an in vitro preparation of the lamprey central nervous system. Brain Res. 300, 357-361. doi: 10.1016/0006-8993(84)90846-1

Mitchell, E. J., McCallum, S., Dewar, D., and Maxwell, D. J. (2016). Corticospinal and reticulospinal contacts on cervical commissural and long descending propriospinal neurons in the adult rat spinal cord; evidence for powerful reticulospinal connections. PLoS One 11:e0155664. doi: 10.1371/journal.pone. 0155664

Mori, S. (1987). Integration of posture and locomotion in acute decebrate cats and in a wake, freely moving cats. Prog. Neurobiol. 28, 161-195. doi: 10.1016/03010082(87)90010-4

Mori, S., Kawahara, K., Sakamoto, T., Aoki, M., and Tomiyama, T. (1982). Setting and resetting of level of postural muscle tone in decerebrate cat by stimulation of brain stem. J. Neurophysiol. 48, 737-748. doi: 10.1152/jn.1982.48.3.737

Mori, S., Matsui, T., Kuze, B., Asanome, M., Nakajima, K., and Matsuyama, K. (1998). Cerebellar-induced locomotion: reticulospinal control of spinal rhythm generating mechanism in cats. Ann. N Y Acad. Sci. 860, 94-105. doi: 10.1111/j. 1749-6632.1998.tb09041.x

Mori, S., Nishimura, H., Kurakami, C., Yamamura, T., and Aoki, M. (1978). Controlled locomotion in the mesencephalic cat: distribution of facilitatory and inhibitory regions within pontine tegmentum. J. Neurophysiol. 41, 1580-1591. doi: 10.1152/jn.1978.41.6.1580

Mori, S., Ohta, Y., Matsuyama, K., and Takukuski, K. (1986). "The setting of postural muscle tone: its importance for behaviors in freely moving intact cats," in Emotions: Neuronal and Chemical Control, ed. Y. Oomura (Basel: Karger), 231-239.

Mouret, J., Delorme, F., and Jouvet, M. (1967). Lesions of the pontine tegmentum field. Compt. Soc. Biol. Fil. 161, 1603-1606.

Noga, B. R., Kettler, J., and Jordan, L. M. (1988). Locomotion produced in mesencephalic cats by injections of putative transmitter substances and antagonists into the medial reticular formation and the pontomedullary locomotor strip. J. Neurosci. 8, 2074-2086. doi: 10.1523/JNEUROSCI.08-0602074.1988

Noga, B. R., Kriellaars, D. J., Brownstone, R. M., Jordan, L. M., Leiras, R., Velo, P., et al. (2003). Mechanism for activation of locomotor centers in the spinal cord by stimulation of the mesencephalic locomotor region mechanism for activation of locomotor centers in the spinal cord by stimulation of the mesencephalic locomotor region. J. Neurophysiol. 90, 1464-1478. doi: $10.1152 /$ jn. 00034.2003

Orlovskii, G. N. (1970). Work of the reticulo-spinal neurons during locomotion. Biofizika 15, 728-737.

Paxinos, G., and Franklin, B. (2008). The Mouse Brain in Sterotaxic Coordinates. 3rd Edn. Oxford: Academic Press.

Paxinos, G., Xu-Feng, H., Sengul, G., and Watson, C. (2012). "Organization of brainstem nuclei," in The Huaman Nervous System, eds J. K. Mai and G. Paxinos (Amsterdam: Elsevier Academic Press), 260-327.
Perreault, M. C., Drew, T., and Rossignol, S. (1993). Activity of medullary reticulospinal neurons during fictive locomotion. J. Neurophysiol. 69, 2232-2247. doi: 10.1152/jn.1993.69.6.2232

Perreault, M. C., and Glover, J. C. (2013). Glutamatergic reticulospinal neurons in the mouse: developmental origins, axon projections, and functional connectivity. Ann. N Y Acad. Sci. 1279, 80-89. doi: 10.1111/nyas.12054

Peterson, B. W. (1984). "The reticulospinal system and its role in the control of movement," in Brainstem Control of Spinal Cord Function, ed. C. D. Barnes (New York, NY: Academic Press), 27-86.

Peterson, B. W., Maunz, R. A., Pitts, N. G., and Mackel, R. G. (1975). Patterns of projection and branching of reticulospinal neurons. Exp. Brain Res. 23, 333-351. doi: 10.1007/bf00238019

Peterson, B. W., Pitts, N. G., Fukushima, K., and Mackel, R. (1978). Reticulospinal excitation and inhibition of neck motoneurons. Exp. Brain Res. 32, 471-489. doi: $10.1007 / \mathrm{bf} 00239548$

Prasada Rao, P. D., Jadhao, A. G., and Sharma, S. C. (1987). Descending projection neurons to the spinal cord of the goldfish, Carassius auratus. J. Comp. Neurol. 265, 96-108. doi: 10.1002/cne.902650107

Rhines, R., and Magoun, H. (1946). Brain stem facilitation of cortical motor response. J. Neurophysiol. 9, 219-229. doi: 10.1152/jn.1946.9.3.219

Riddle, C. N., Edgley, S. A., and Baker, S. N. (2009). Direct and indirect connections with upper limb motoneurons from the primate reticulospinal tract. J. Neurosci. 29, 4993-4999. doi: 10.1523/JNEUROSCI.3720-08.2009

Roseberry, T. K., Lee, A. M., Lalive, A. L., Wilbrecht, L., Bonci, A., and Kreitzer, A. C. (2016). Cell-type-specific control of brainstem locomotor circuits by basal ganglia. Cell 164, 526-537. doi: 10.1016/j.cell.2015. 12.037

Rothwell, J. C. (2006). The startle reflex, voluntary movement, and the reticulospinal tract. Suppl. Clin. Neurophysiol. 58, 223-231. doi: 10.1016/s1567424x(09)70071-6

Saper, C. B., Fuller, P. M., Pedersen, N. P., Lu, J., and Scammell, T. E. (2010). Sleep state switching. Neuron 68, 1023-1042. doi: 10.1016/j.neuron.2010. 11.032

Sasaki, S., Isa, T., Pettersson, L. G., Alstermark, B., Naito, K., Yoshimura, K., et al. (2004). Dexterous finger movements in primate without monosynaptic corticomotoneuronal excitation. J. Neurophysiol. 92, 3142-3147. doi: $10.1152 /$ jn. 00342.2004

Sastre, J. P., Sakai, K., and Jouvet, M. (1981). Are the gigantocellular tegmental field neurons responsible for paradoxical sleep? Brain Res. 229, 147-161. doi: 10.1016/0006-8993(81)90752-6

Schenck, C. H., and Mahowald, M. W. (1995). A polysomnographically documented case of adult somnambulism with long-distance automobile driving and frequent nocturnal violence: parasomnia with continuing danger as a noninsane automatism? Sleep 18, 765-772. doi: 10.1093/sleep/ 18.9.765

Schepens, B. (2004). Independent and convergent signals from the pontomedullary reticular formation contribute to the control of posture and movement during reaching in the cat. J. Neurophysiol. 92, 2217-2238. doi: $10.1152 /$ jn. 01189.2003

Schmidt, B. J., and Jordan, L. M. (2000). The role of serotonin in reflex modulation and locomotor rhythm production in the mammalian spinal cord. Brain Res. Bull. 53, 689-710. doi: 10.1016/s0361-9230(00)00402-0

Shapovalov, A. (1972). Evolution of neuronal systems of suprasegmental motor control. Neurophysiology 4, 346-359. doi: 10.1007/bf01063005

Sherrington, C. S. (1898). Decerebrate rigidity and reflex coordination of movements. J. Physiol. 22, 319-332. doi: 10.1113/jphysiol.1898.sp000697

Shik, M. L., Severin, F. V., and Orlovskii, G. N. (1966). Control of walking and running by means of electrical stimulation of the midbrain. Biofizika 11, 659-666.

Shimamura, M., and Kogure, I. (1983). Discharge patterns of reticulospinal neurons corresponding with quadrupedal leg movements in thalamic cats. Brain Res. 260, 27-34. doi: 10.1016/0006-8993(83)90761-8

Shimamura, M., Kogure, I., and Wada, S. (1980). Three types of reticular neurons involved in the spinobulbo-spinal reflex of cats. Brain Res. 186, 99-113. doi: 10.1016/0006-8993(80)90258-9

Shimamura, M., Kogure, I., and Wada, S. I. (1982). Reticular neuron activities associated with locomotion in thalamic cats. Brain Res. 231, 51-62. doi: 10.1016/0006-8993(82)90006-3 
Siegel, J. M., Nienhuis, R., Wheeler, R. L., McGinty, D. J., and Harper, R. M. (1981). Discharge pattern of reticular formation unit pairs in waking and REM sleep. Exp. Neurol. 74, 875-891. doi: 10.1016/0014-4886(81)90260-0

Sillar, K. T., Picton, L. D., and Heitler, W. J. (2016). The Neuroethology of Predation and Escape. 1st Edn. Oxford: Wiley-Blackwell.

Sinnamon, H. M. (1993). Preoptic and hypothalamic neurons and the initiation of locomotion in the anesthetized rat. Prog. Neurobiol. 41, 323-344. doi: 10.1016/0301-0082(93)90003-b

Sinnamon, H. M., and Stopford, C. K. (1987). Locomotion elicited by lateral hypothalamic stimulation in the anesthetized rat does not require the dorsal midbrain. Brain Res. 402, 78-86. doi: 10.1016/0006-8993(87) 91049-3

Sivertsen, M. S., Perreault, M. C., and Glover, J. C. (2016). Pontine reticulospinal projections in the neonatal mouse: internal organization and axon trajectories. J. Comp. Neurol. 524, 1270-1291. doi: 10.1002/cne.23904

Skinner, R. D., and Garcia-Rill, E. (1984). The mesencephalic locomotor region (MLR) in the rat. Brain Res. 323, 385-389. doi: 10.1016/0006-8993(84) 90319-6

Sprague, J. M., and Chamber, W. W. (1954). Control of posture by reticular formation and cerebellum in the intact, anesthetized and unanesthetized and in the decerebrated cat. Am. J. Physiol. 176, 52-64. doi: 10.1152/ajplegacy.1953.176.1.52

Stecina, K. (2017). Midbrain stimulation-evoked lumbar spinal activity in the adult decerebrate mouse. J. Neurosci. Methods 288, 99-105. doi: 10.1016/j.jneumeth. 2017.06.015

Steeves, J. D., and Jordan, L. M. (1980). Localization of a descending pathway in the spinal cord which is necessary for controlled treadmill locomotion. Neurosci. Lett. 20, 282-288. doi: 10.1016/0304-3940(80)90161-5

Steeves, J. D., Sholomenko, G. N., and Webster, D. M. S. (1987). Stimulation of the pontomedullary reticular formation initiates locomotion in decerebrate birds. Brain Res. 401, 205-212. doi: 10.1016/0006-8993(87)91406-5

Szokol, K., Glover, J. C., and Perreault, M.-C. (2011). Organization of functional synaptic connections between medullary reticulospinal neurons and lumbar descending commissural interneurons in the neonatal mouse. J. Neurosci. 31, 4731-4742. doi: 10.1523/JNEUROSCI.5486-10.2011

Szokol, K., Glover, J. C., Perreault, M.-C., and Perreault, M.-C. (2008). Differential origin of reticulospinal drive to motoneurons innervating trunk and hindlimb muscles in the mouse revealed by optical recording. J. Physiol. 58621, 5259-5276. doi: 10.1113/jphysiol.2008.158105

Takakusaki, K., Chiba, R., Nozu, T., and Okumura, T. (2016). Brainstem control of locomotion and muscle tone with special reference to the role of the mesopontine tegmentum and medullary reticulospinal systems. J. Neural Transm. (Vienna) 123, 695-729. doi: 10.1007/s00702-015-1475-4
Takakusaki, K., Kohyama, J., Matsuyama, K., and Mori, S. (1993a). Synaptic mechanisms acting on lumbar motoneurons during postural augmentation induced by serotonin injection into the rostral pontine reticular formation in decerebrate cats. Exp. Brain Res. 93, 471-482. doi: 10.1007/bf002 29362

Takakusaki, K., Matsuyama, K., Kobayashi, Y., Kohyama, J., and Mori, S. (1993b). Pontine microinjection of carbachol and critical zone for inducing postural atonia in reflexively standing decerebrate cats. Neurosci. Lett. 153, 185-188. doi: 10.1016/0304-3940(93)90318-f

Valencia Garcia, S., Brischoux, F., Clément, O., Libourel, P.-A., Arthaud, S., Lazarus, M., et al. (2018). Ventromedial medulla inhibitory neuron inactivation induces REM sleep without atonia and REM sleep behavior disorder. Nat. Commun. 9:504. doi: 10.1038/s41467-017-02761-0

Valls-Solé, J., Rothwell, J. C., Goulart, F., Cossu, G., and Muñoz, E. (1999). Patterned ballistic movements triggered by a startle in healthy humans. J. Physiol. 516, 931-938. doi: 10.1111/j.1469-7793.1999.0931u.x

Wilson, V. J., and Yoshida, M. (1969). Comparison of effects of stimulation of Deiters' nucleus and medial longitudinal fasciculus on neck, forelimb and hindlimb motoneurons. J. Neurophysiol. 32, 743-758. doi: 10.1152/jn.1969. 32.5.743

Wilson, V. J., Yoshida, M., and Schor, R. H. (1970). Supraspinal monosynaptic excitation and inhibition of thoracic back motoneurons. Exp. Brain Res. 11, 282-295. doi: 10.1007/bf01474387

Yasargil, G. M., and Sandri, C. (1990). Topography and ultrastructure of commissural interneurons that may establish reciprocal inhibitory connections of the Mauthner axons in the spinal cord of the tench, Tinca tinca L. J. Neurocytol. 19, 111-126. doi: 10.1007/bf01188443

Zaaimi, B., Edgley, S. A., Soteropoulos, D. S., and Baker, S. N. (2012). Changes in descending motor pathway connectivity after corticospinal tract lesion in macaque monkey. Brain 135, 2277-2289. doi: 10.1093/brain/aws115

Zottoli, S. J. (1977). Correlation of the startle reflex and Mauthner cell auditory responses in unrestrained goldfish. J. Exp. Biol. 66, 243-254.

Conflict of Interest Statement: The authors declare that the research was conducted in the absence of any commercial or financial relationships that could be construed as a potential conflict of interest.

Copyright (C) 2018 Brownstone and Chopek. This is an open-access article distributed under the terms of the Creative Commons Attribution License (CC BY). The use, distribution or reproduction in other forums is permitted, provided the original author(s) and the copyright owner are credited and that the original publication in this journal is cited, in accordance with accepted academic practice. No use, distribution or reproduction is permitted which does not comply with these terms. 